\title{
Aboriginal and Torres Strait Islander health checks: sociodemographic characteristics and cardiovascular risk factors
}

\author{
Danielle C Butler ${ }^{a}$, Jason Agostino ${ }^{a, b}$, Ellie Paige ${ }^{a}$, Rosemary J Kordaa, \\ Kirsty A Douglas ${ }^{b}$, Vicki Wade ${ }^{c}$ and Emily Banks ${ }^{a}$
}

a National Centre for Epidemiology and Population Health, Australian National University, Canberra, ACT

b Academic Unit of General Practice, Australian National University, Canberra, ACT

c Menzies School of Health Research, Charles Darwin University, NT, Australia

d Corresponding author: danielle.butler@anu.edu.au

\section{Article history}

Publication date: February 2021

Citation: Butler DC, Agostino J, Paige E, Korda RJ, Douglas KA, Wade V, Banks E. Aboriginal and Torres Strait Islander health checks: sociodemographic characteristics and cardiovascular risk factors. Public Health Res Pract. 2021; Online early publication. https://doi.org/10.17061/ phrp31012103

\section{Key points}

- Over a 2-year period, one-third of Aboriginal and Torres Strait Islander people in the study population received a Medicare-funded health check

- Those with cardiovascular disease (CVD) risk factors and prior CVD were more likely to have received a health check, while those with better self-rated health and infrequent users of general practice services were less likely to have done so

- Increasing participation in health checks may improve identification and management of Aboriginal and Torres Strait Islander people at high risk of primary and secondary CVD events

\section{Abstract}

Objective: To quantify Aboriginal and Torres Strait Islander health check claims in Australian adults in relation to sociodemographic and health characteristics, including prior cardiovascular disease (CVD) and CVD risk factors.

Methods: The study involved analysis of baseline data (2006-2009) from the Sax Institute's 45 and Up Study, involving 1753 Aboriginal and Torres Strait Islander adults in New South Wales, Australia, linked to Medicare Benefits Schedule (MBS) hospital and death data (to December 2015). The outcome was a claim for receiving a Medicare-funded Health Assessment for Aboriginal and Torres Strait Islander People (MBS item 715) in the 2 years before December 2015. Logistic regression was used to estimate odds ratios (ORs) for receiving a health check in relation to sociodemographic and health characteristics.

Results: One-third (32\%) of participants received at least one Medicarefunded health check in the 2-year period. The probability of receiving a health check was higher for women than men (adjusted OR 1.47; 95\% Cl 1.18, 1.84), for those with lowest education than for those with highest education (OR 1.58; $\mathrm{Cl} 1.11,2.24$ ), for those in a regional area (OR 1.56; Cl 1.22, 2.01) or remote area $(\mathrm{OR} 2.38 ; \mathrm{Cl} 1.8,3.16)$ than for those in major cities, for those with prior CVD than for those without (OR 1.80; Cl 1.42, 2.27), for those with CVD risk factors than for those without (adjusted OR between 1.28 and 2.28, depending on risk factor), for those with poor self-rated health than for those with excellent self-rated health (OR 3.15; Cl 1.76, 6.65) and for those with more than 10 visits to a general practitioner (GP) per year than for those with 0-2 visits (OR 33.62; Cl 13.45, 84.02). Additional adjustment for number of GP visits or self-rated health substantially attenuated ORs for prior CVD and most CVD risk factors. When mutually adjusted, use of GP services and poorer selfrated health remained strongly associated with receiving a health check. 
Conclusions: Aboriginal and Torres Strait Islander people with the greatest healthcare need and at highest risk of CVD were more likely to receive a health check; however, a significant proportion of those who were eligible had not received this preventive care intervention. Findings indicate that there is greater potential for the use of health checks (MBS item 715) in improving identification and management of Aboriginal and Torres Strait Islander people at high risk of CVD, potentially preventing future CVD events.

\section{Introduction}

Cardiovascular disease (CVD) is the largest contributor to Aboriginal and Torres Strait Islander mortality. ${ }^{1}$ Although age-standardised mortality rates due to CVD have fallen by $40 \%$ over the past few decades, CVD still accounts for one-quarter of Aboriginal and Torres Strait Islander deaths overall and $21 \%$ of all premature years of life lost. ${ }^{1}$ Colonisation and the resultant ongoing disempowerment, disruption of connection to culture and land, and intergenerational trauma are likely to have played major roles in current levels of social, emotional and physical wellbeing of Aboriginal and Torres Strait Islander people ${ }^{2}$, including CVD mortality. CVD events are highly preventable, including through effective management of risk. Absolute CVD risk assessment and management are recommended nationally and internationally ${ }^{3,4}$; however, around half of Aboriginal and Torres Strait Islander people at high absolute CVD risk are not receiving guidelinerecommended therapy.

One mechanism for improving management of preventive healthcare needs and CVD risk assessment is the Medicare Benefits Schedule (MBS) Health Assessment for Aboriginal and Torres Strait Islander People (MBS item number 715, herein referred to as a health check). All Australians who identify as Aboriginal or Torres Strait Islander are eligible to access this annual health check, which is funded by the Australian Government via Medicare, and involves an assessment by a general practitioner (GP) of a patient's physical, psychological and social wellbeing. ${ }^{7}$ Studies conducted in mostly remote Aboriginal and Torres Strait Islander community settings have found that those who have had a health check are more likely to have their absolute CVD risk determined ${ }^{6,8}$, and that this is associated with improved care and a reduction in subsequent estimated CVD risk. ${ }^{9}$ Limited international evidence suggests that health checks in general can lead to increased new diagnoses (including CVD risk factors) ${ }^{10}$ and to improvements in intermediate health outcomes, such as blood pressure and cholesterol levels. ${ }^{11}$

Although the number of annual health checks undertaken has increased since 2010-11, Medicare data show that only an estimated $29 \%$ of the eligible population had health checks in the 2017-18 financial year. ${ }^{12}$ The Australian Government is seeking to more than double the proportion of the eligible population receiving Aboriginal and Torres Strait Islander health checks. ${ }^{13}$
However, action to achieve this target is constrained by limited data on who receives health checks and who is missing out. Recent national estimates of health check claims have been reported by age, sex and remoteness. ${ }^{12}$ Individual-level studies have examined delivery of preventive services that are typically part of a health check ${ }^{14,15}$, primarily in remote Aboriginal and Torres Strait Islander-specific health services. No studies have examined in detail the individual sociodemographic and health characteristics of those receiving health checks across a range of settings.

The aim of this study was to quantify claims for the Aboriginal and Torres Strait Islander health check (MBS item 715) in a 2-year period among Aboriginal and Torres Strait Islander adults from the general population of New South Wales (NSW), Australia, in relation to sociodemographic and health characteristics, including prior CVD and CVD risk factors.

\section{Methods}

\section{Setting}

Approximately 649000 Aboriginal and Torres Strait Islander people live in Australia, comprising $2.8 \%$ of the total population. ${ }^{16}$ Just over one-third of Aboriginal and Torres Strait Islander people live in NSW - the largest population of all states and territories in Australia. ${ }^{16}$ In NSW, $85.5 \%$ of Aboriginal and Torres Strait Islander people live in urban areas. ${ }^{16}$

\section{Study population}

The Sax Institute's 45 and Up Study is a large prospective cohort study of people aged 45 years and older residing in NSW. Participants were randomly sampled from the enrolment database of Services Australia (formerly the Department of Human Services and Medicare Australia), with over-sampling by a factor of two of individuals aged 80 years and older and people residing in rural areas. Participants enrolled in the study by completing a baseline questionnaire, distributed between 2006 and 2009, and providing consent for 5-yearly questionnaires and linkage to routinely collected health data. Approximately $10 \%$ of the total NSW population aged 45 years and older was included in the study, with a response rate of around $18 \% .{ }^{17}$ The study design and details of the questionnaire are reported elsewhere. ${ }^{17-19}$ 
For this study, we included participants who self-identifed as Aboriginal and/or Torres Strait Islander on the baseline questionnaire ( $n=1753 / 266512$ participants [0.7\%], 2016 data release).

\section{Data}

Sociodemographic and health variables were derived from the self-reported baseline questionnaire. Data from the questionnaire were linked to MBS claims data (1 January 2003 - 31 December 2015) provided by the Department of Human Services, the NSW Admitted Patient Data Collection (APDC, 1 July 2001 - 30 June 2017), and data from the NSW Registry of Births, Deaths and Marriages (RBDM) and the National Death Index (NDI). Probabilistic linkage was performed by the Centre for Health Record Linkage (CHeReL) for APDC and NSW RBDM data. Quality assurance data on the CHeReL data linkage show false positive and negative rates of $<0.5 \%$ and $<0.1 \%$, respectively. ${ }^{19} \mathrm{NDI}$ data were linked by the Australian Institute of Health and Welfare. ${ }^{20}$ Further details can be found in the supplementary file (available from: hdl.handle.net/1885/220008).

\section{Variable definition}

The outcome studied was a claim for an Aboriginal and Torres Strait Islander health check (yes/no), defined as at least one MBS item 715 claim during the period 1 January 2014 - 31 December 2015, based on linked MBS records.

Participant characteristics were derived from the 45 and Up Study baseline questionnaire, unless otherwise indicated, and included the following:

- Sociodemographic variables - age, sex, marital status, highest educational qualification and remoteness of residence, as shown in Table 1. Remoteness was categorised based on postcode of residence mean Acessibility and Remoteness Index of Australia score. ${ }^{21}$

- Self-reported CVD risk factors - smoking status, body mass index (BMI), alcohol consumption, physical activity, doctor-diagnosed diabetes, doctor-diagnosed high blood pressure and/or treatment for high blood pressure, and treatment for high blood cholesterol. BMI was categorised as not overweight or obese $(<25$ $\left.\mathrm{kg} / \mathrm{m}^{2}\right)$, overweight and not obese $\left(25\right.$ to $\left.<30 \mathrm{~kg} / \mathrm{m}^{2}\right)$ or obese $\left(\geq 30 \mathrm{~kg} / \mathrm{m}^{2}\right)$. Physical activity was determined using items from the Active Australia Survey ${ }^{22}$, with sufficient activity defined as at least 150 minutes of physical activity per week over at least five sessions per week

- Prior CVD - any CVD (self-reported doctor-diagnosed heart disease or stroke; self-reported treatment for heart attack, angina or other heart disease; or hospitalisation for any major CVD condition); and ischaemic heart disease (IHD) or cerebrovascular disease (limited to self-reported doctor-diagnosed stroke, self-reported treatment or hospitalisation for heart attack, angina, IHD, cerebrovascular disease or transient ischaemic attack only). For these variables, hospitalisations were derived from linked hospital data. They included those before the study period (1 January 2014 for main analyses) and until 5 years before enrolment to the 45 and Up Study. See the supplementary file for a full list of ICD-10 codes included (hdl.handle.net/1885/220008)

- GP visits - total number of GP attendances in the follow-up period, derived from linked MBS data, categorised as low (0-2 visits per year), medium (310 visits per year) and high ( $\geq 11$ visits per year) (see appendix in supplementary files for a list of included item numbers: hdl.handle.net/1885/220008)

- Self-rated health status - excellent, very good, good, fair or poor.

\section{Statistical analysis}

Participants were followed up for 2 years from 1 January 2014. Individuals were excluded from the analysis if they had an invalid death date $(n=187)$ or died in the follow-up period ( $n=194$, determined from linked death data). Frequencies and proportions were calculated for the sample according to participant characteristics, for the total sample and by claim for a health check. Logistic regression was used to estimate odds ratios (ORs) and 95\% confidence intervals (95\% $\mathrm{Cl})$ for receiving a health check in relation to participant characteristics, sequentially adjusting for age and sex; then adding sociodemographic characteristics, CVD risk factors and prior CVD; then separately for annual number of GP visits and self-rated health. The significance of the addition of an explanatory variable to the model was determined using the Wald joint test of significance.

Analyses were undertaken using Stata (College Station, Texas: StataCorp; Version 14.1) in the Secure Unified Research Environment, a secure remote-access computer facility for analysis of linked data.

\section{Sensitivity analyses}

Supplementary analyses were run for two alternative follow-up periods (1 January 2010 - 31 December 2011 and 1 January 2012 - 31 December 2013), as patterns of association may differ between years. In addition, we repeated analyses, to include those who received a health check but did not identify as Aboriginal or Torres Strait Islander in the baseline survey $(24.8 \%$ of those who had at least one MBS item 715 recorded in the linked MBS dataset), and those who died in the follow-up period - in both instances, these participants may differ in their sociodemographic and health risk profile from other participants. 
Table 1. Participant characteristics for total sample

\begin{tabular}{lcc}
\hline & Study participants, $n$ & $\%$ \\
\hline Total Sample & 1753 & 100 \\
\hline Sociodemographic characteristic & \\
Age (years) & \\
$45-49$ & 405 & 23.1 \\
$50-54$ & 451 & 25.7 \\
$55-59$ & 346 & 19.7 \\
$60-64$ & 238 & 13.6 \\
$65-69$ & 159 & 9.1 \\
$\geq 70$ & 154 & 8.8 \\
\hline
\end{tabular}

\section{Sex}

Male

733

41.8

Female

1020

58.2

\section{Marital status}

\begin{tabular}{lcc} 
Single & 636 & 36.3 \\
Partnered & 1098 & 62.6 \\
\hline
\end{tabular}

\section{Education}

No school certificate

482

School certificate,

973

27.5

55.5

trade or diploma

249

14.2

Degree or higher
Region of residence

$\begin{array}{lll}\text { Major city } & 672 & 38.3 \\ \text { Inner regional } & 669 & 38.2 \\ \text { Outer regional/ } & 383 & 21.9\end{array}$

remote

\section{Cardiovascular disease risk factors}

\section{Smoking status}

\begin{tabular}{lll} 
Never & 732 & 41.8 \\
Past & 636 & 36.3 \\
Current & 376 & 21.5 \\
\hline
\end{tabular}

\section{Alcohol consumption (drinks per week)}

\begin{tabular}{lll}
0 & 767 & 43.8 \\
1 to 14 & 666 & 38.0 \\
$\geq 15$ & 241 & 13.8 \\
\hline $\mathbf{B M I}\left(\mathbf{k g} / \mathbf{m}^{2}\right)$ & & \\
$<25$ & 379 & 21.6 \\
$25-29$ & 588 & 33.5 \\
$\geq 30$ & 584 & 33.3 \\
\hline Physical activity & & \\
Insufficient & 596 & 34.0 \\
Sufficient & 987 & 56.3 \\
\hline Diabetes & & \\
No & 1487 & 84.8 \\
Yes & 266 & 15.2 \\
\hline
\end{tabular}

\begin{tabular}{lcc}
\hline & Study participants, $n$ & $\%$ \\
\hline High blood pressure & & \\
No & 1069 & 61.0 \\
Yes & 684 & 39.0 \\
\hline High blood cholesterol & & \\
No & 1470 & 83.9 \\
Yes & 283 & 16.1 \\
\hline
\end{tabular}

Prior IHD or cerebrovascular disease

\begin{tabular}{lcc} 
No & 1406 & 80.2 \\
Yes & 347 & 19.8 \\
\hline Prior CVD (any) & & \\
No & 1173 & 66.9 \\
Yes & 580 & 33.1
\end{tabular}

\section{General health status}

\section{Self-rated health}

\begin{tabular}{lcc} 
Excellent & 155 & 8.8 \\
Very good & 461 & 26.3 \\
Good & 594 & 33.9 \\
Fair & 350 & 20.0 \\
Poor & 103 & 5.9 \\
\hline GP use (visits/year) & & \\
$0-2$ & 155 & 8.8 \\
$3-10$ & 813 & 46.4 \\
$\geq 11$ & 785 & 44.8
\end{tabular}

$\mathrm{BMI}=$ body mass index; CVD = cardiovascular disease; $\mathrm{GP}=$ general practitioner; IHD = ischaemic heart disease

Notes: $n=$ total number of participants in that category; $\%=$ percentage of participants in that category of the total sample (complete data, including missing responses). Columns sum to $100 \%$, including missing participants and missing responses. Proportions missing for each variable $(\%(n))$ were as follows: marital status $1.2(19)$, education 2.8 (49), residence 1.7 (29), smoking 0.5 (79), alcohol consumption (drinks per week) 4.5 (79), BMI 11.5 (202), physical activity 9.7 (170), self-rated health $5.13(90)$.

\section{Ethics approval}

Ethics approval for the 45 and Up Study is provided by the University of NSW Human Research Ethics Committee (reference 2010/05/389). For this study, ethics approval was provided by the Aboriginal Health and Medical Research Council of NSW (reference 1006/14) and the Australian National University Human Research Ethics Committee (reference 2012/504). The 45 and Up Study participants consented specifically to linkage of MBS data, which is performed under approvals from the ethics committees of the Australian Government Department of Human Services and the Australian Government Department of Health. 


\section{Results}

\section{Sample characteristics}

The final study population of 1753 Aboriginal and/or Torres Strait Islander adults had a mean age of 57 years. Of these people, $58 \%$ were female and $22 \%$ lived in remote areas (Table 1). Overall, $67 \%$ were overweight or obese, $22 \%$ were current smokers, $15 \%$ reported doctordiagnosed diabetes, 39\% reported doctor-diagnosed high blood pressure or treatment for high blood pressure, and $16 \%$ reported treatment for high blood cholesterol. Almost $20 \%$ had prior ischaemic heart disease or cerebrovascular disease, and 33\% had any CVD. Most participants (91\%) made at least three GP visits per year in the follow-up period, with $45 \%$ making more than 10 visits per year; $2 \%$ had no record of an MBS claim for a GP service.

\section{Association between sociodemographic and health characteristics for receipt of a health check}

Overall, $32 \%$ of participants had received at least one health check in the 2-year period from 1 January 2014 to 31 December 2015.

After adjusting for age and sex, most characteristics were associated with receiving a health check, and results did not change materially after adjusting for all sociodemographic variables (Figure 1). The probability of receiving a health check was higher for women than men (adjusted OR 1.47; 95\% Cl 1.18, 1.84), for those with lowest versus highest education (OR 1.58; 95\% $\mathrm{Cl} 1.11,2.24)$, and for those living in inner regional areas (OR 1.56; 95\% Cl 1.22, 2.01) or outer regional/ remote areas (OR 2.38; 95\% Cl 1.8, 3.16) versus major cities. The probability of receiving a health check was also higher in those with any prior CVD (OR 1.80; 95\% $\mathrm{Cl} 1.42,2.27)$, or prior IHD or cerebrovascular disease, than in those without (OR 1.65; 95\% Cl 1.25, 2.18); and in those with CVD risk factors, including smoking, overweight and obesity, diabetes and high blood pressure, compared with those without these risk factors (OR 1.28-2.28) (Figure 2). The odds of receiving a health check among those with poor self-rated health were 3.15 times those of participants with excellent health (95\% $\mathrm{Cl} 1.76,6.65)$. People with $\geq 11 \mathrm{MBS}$ GP visits per year were 33.62 times more likely to receive a health check $(95 \% \mathrm{Cl} 13.45,84.02)$ than those with 0-2 GP visits per year (Figure 2).

Additional adjustment - for either annual number of MBS GP services or self-rated health - substantially attenuated the ORs for receiving a health check in relation to treatment for high blood pressure, high blood cholesterol and prior CVD (any CVD or IHD/ cerebrovascular disease), suggesting that use of services and general health status explained at least part, if not all, of the relationship of these risk factors and health conditions with receiving health checks. Use of GP services and poorer self-rated health remained strongly associated with receiving a health check (see online supplementary materials: hdl.handle.net/1885/220008).

Figure 1. Receipt of Health Assessment for Aboriginal and Torres Strait Islander People (MBS item 715) by sociodemographic characteristics: proportions, odds ratios and 95\% confidence intervals

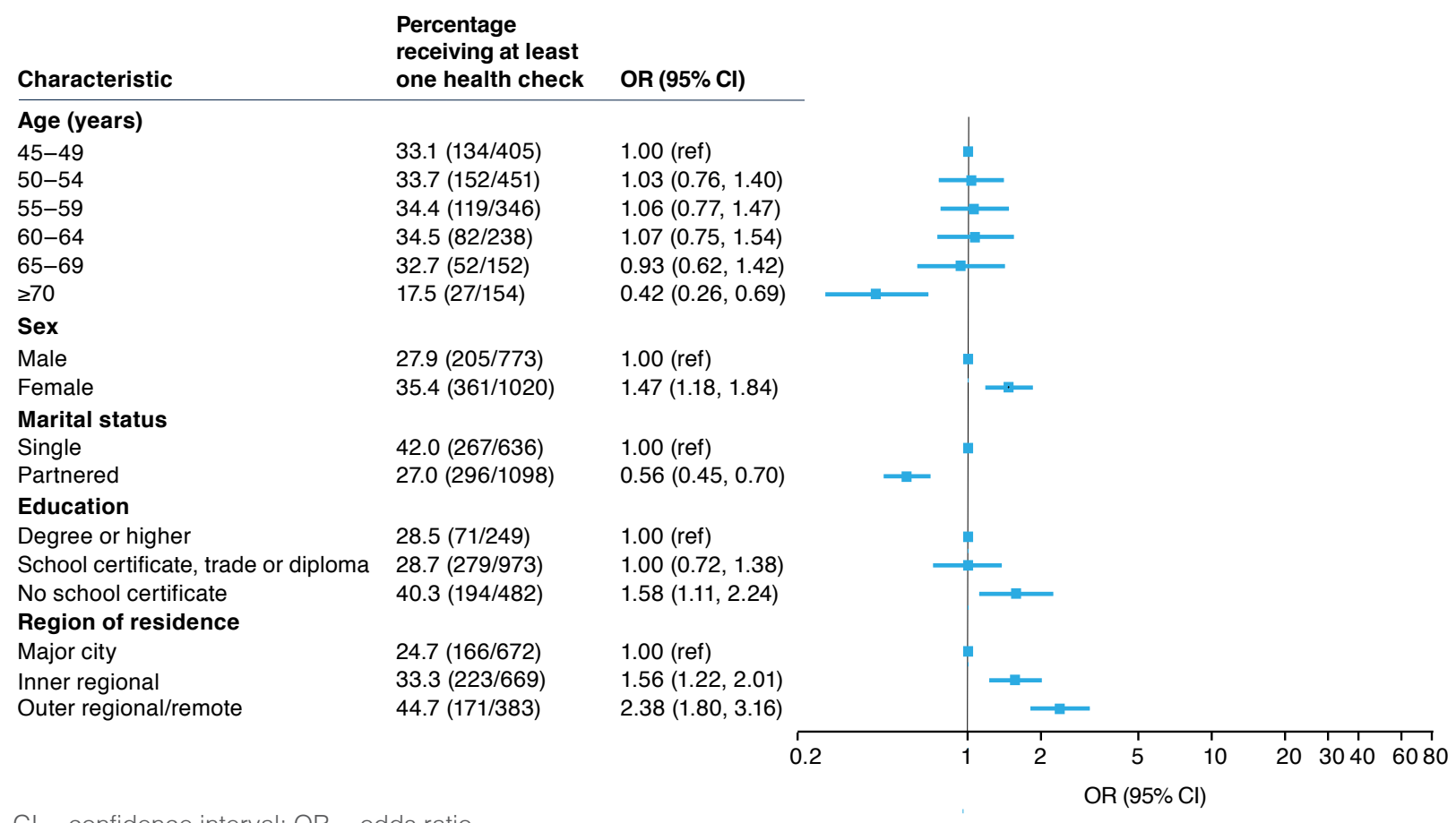


Figure 2. Receipt of Health Assessment for Aboriginal and Torres Strait Islander People (MBS item 715) by CVD and CVD risk factors, general health status and GP use: proportions, odds ratios and 95\% confidence intervals

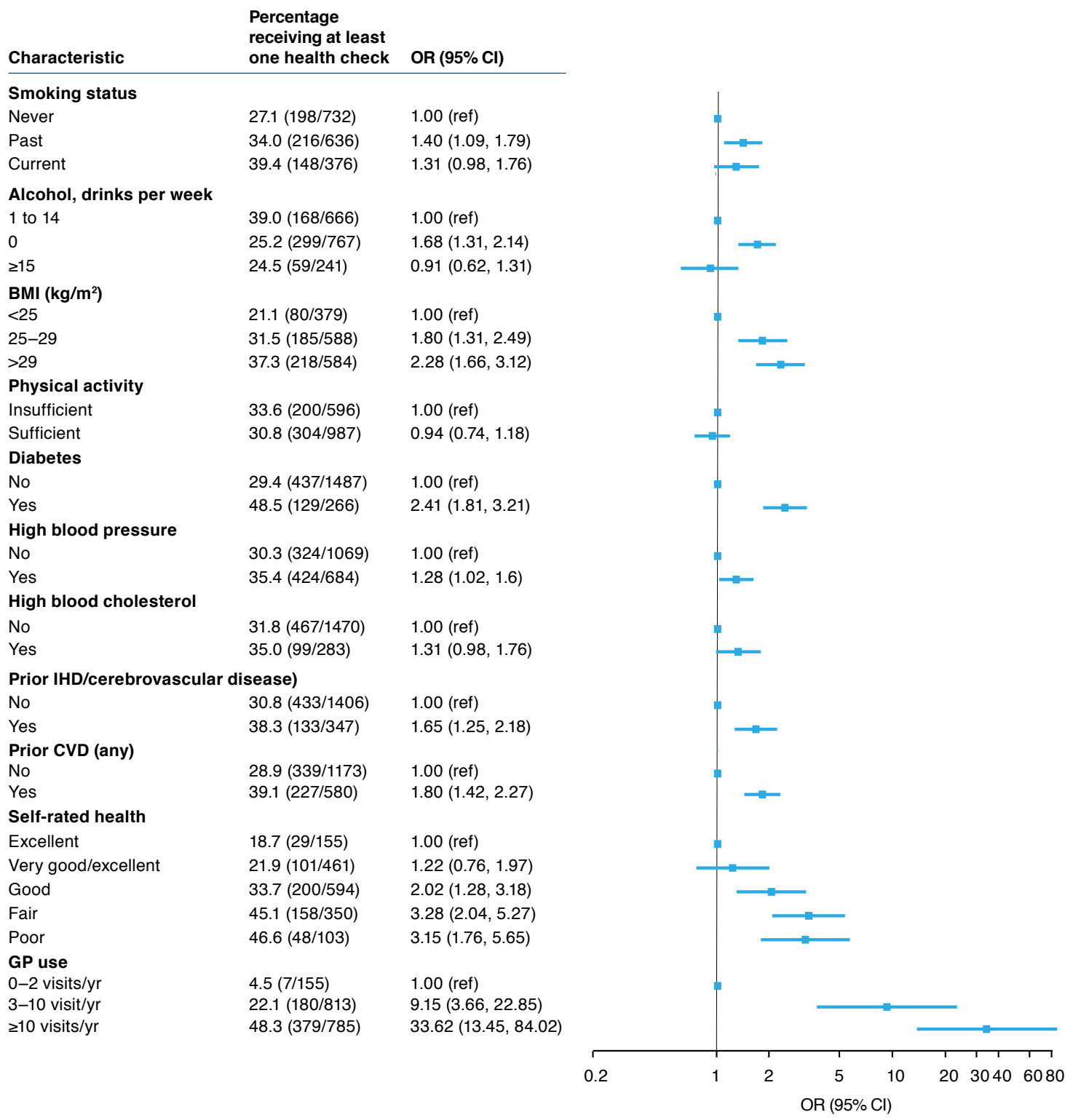

$\mathrm{BMI}=$ body mass index; $\mathrm{Cl}=$ confidence interval; $\mathrm{CVD}=$ cardiovascular disease; $\mathrm{GP}=$ general practitioner; IHD = ischaemic heart

Results of sensitivity analyses did not differ materially from the main results (see online supplementary materials: hdl.handle.net/1885/220008).

\section{Discussion}

Approximately one-third of participants received a Medicare-funded health check over a 2-year period in this large population-based study of Aboriginal and Torres Strait Islander adults living in NSW. Importantly, those who were disadvantaged, lived remotely, had CVD risk factors or established CVD, and had poorer self-rated health were the most likely to receive a health check.

The frequency of accessing GP care was an important factor in the likelihood of people receiving a health check, possibly reflecting higher use by those who were unwell and had a greater need for health services generally. This is supported by the finding that those with poorer self-rated health were more likely to have a claim for MBS item 715. These factors at least partly explained the observed relationship of CVD risk factors and prior CVD with receiving health checks. Living in more remote areas was also strongly associated with receiving health checks. As seen in previous studies ${ }^{8,14}$, area- and/or practice-level factors might relate to whether or not a person receives preventive care (such as a health check).

The overall rate of claims for health checks reported here is similar to estimates nationally in $2017-18^{12}$ and for the 2015-16 NSW population aged 55 years and older (36.2\%). ${ }^{23}$ The finding that women, people living in more remote areas and people with greater use of health services were more likely to receive a health check is consistent with previous Australian studies. ${ }^{12,24}$ 
Recently, it has been reported that areas with large Aboriginal and Torres Strait Islander populations tend to have high uptake of health checks, as well as high rates of potentially preventable hospitalisations and avoidable deaths. ${ }^{25}$ Our finding that those with poorer health and at highest risk of CVD were more likely to receive a health check may partly explain this observation. Further analysis at the individual level is required to clarify this. In contrast to the current findings, international studies of the general population have reported that people who are healthier, with fewer chronic conditions or CVD risk factors, or have a higher socioeconomic position are more likely to have a health check. ${ }^{26}$ These differences may be partly related to differences in study populations or targeting of health checks (using mailed invitations in other countries). In Australia, where health checks are part of usual general practice care and attract specific remuneration for the provider, it is possible that they are more likely to be received by those with more complex healthcare needs.

The uptake of health checks remains a focus of the Australian Government, with a target of more than $60 \%$ coverage of the Aboriginal and Torres Strait Islander adult population by 2023. ${ }^{13}$ Although coverage in 2017-18 is not on track to meet this target, our study found that people with the greatest need were more likely to have had a claim for a health check. At the same time, for health checks to be an effective mechanism for increasing preventive health activities, including absolute CVD risk assessment, a substantial proportion of the population must regularly receive one. Even among frequent users of general practice in our study, fewer than half had a received a health check in the recommended 2-year period. Further, an effective approach to screening and primary prevention needs to reach those who feel well. Those with better self-rated health and/or infrequent users of GP services were less likely than others to receive a health check. These groups are important targets for CVD risk assessment to prevent CVD events.

Increasing health check coverage requires that known barriers are addressed. Studies identify that contributors to low uptake are low levels of identification of Aboriginal and Torres Strait Islander patients, staff concerns about invasive and potentially culturally inappropriate questions, and poor organisational supports for delivery of health assessments. ${ }^{24,27,28}$ Effective implementation of known successful strategies, such as adequate time, allocation of appropriately trained staff to assist and supportive clinical information systems, is key to facilitating uptake of health checks in the broader general practice setting. ${ }^{24}$ Work by the National Aboriginal Community Controlled Health Organisation and the Royal Australian College of General Practitioners aims to address some of these barriers by better aligning the Medicare requirements for MBS item 715 with clinical practice guidelines and highlighting the characteristics of quality health checks, which emphasise patient-centred care. ${ }^{29}$ In addition, addressing known barriers to current low levels of follow-up care (e.g. review of abnormal results, ongoing management and referral) $)^{24,30}$ will be essential for preventing further CVD events.

The Australian Government introduced a Medicare item specifically targeting absolute CVD risk assessment (MBS item 699 and 77) for use across the general population in April 2019. Given that this item cannot be claimed within a year of MBS item 715 and has a substantially lower rebate, it seems unlikely that there will be significant uptake among Aboriginal and Torres Strait Islander people.

This study is the largest to date examining health check claims for a range of personal and health characteristics, across a variety of settings. To our knowledge, it is also the only population-based study using individual data. Previous individual-level studies were restricted to those attending health services, where individuals are known to be sicker and findings are not necessarily generalisable. ${ }^{6,8,9}$ Further, linkage to hospitalisation data provided independent robust data on prior CVD.

The study focuses on the NSW population and those who are aged 45 years and older; patterns of association may differ for other age groups or in other settings. Cohort studies are not designed to be representative, but internal comparisons from cohort studies remain valid and broadly generalisable. ${ }^{18}$ Caution should be applied when interpreting the absolute proportions of health assessment received. As is common to all survey-based studies, these data are potentially subject to self-report bias. However, the impact on the patterns observed is likely to be minimal.

\section{Conclusion}

This study is the first detailed exploration of the sociodemographic and health characteristics, and CVD risk factors among those receiving a Medicare-funded health check in a large, population-based Aboriginal and Torres Strait Islander cohort across a range of regions and health services. The findings are encouraging, in that those with the poorest health and those at most risk of CVD, and hence those who would most benefit from healthcare, were more likely to receive a health check. However, for health checks to be an effective mechanism for preventing CVD, ongoing effort is needed to increase coverage among all Aboriginal and Torres Strait Islander people, particularly among those who feel well and access services less frequently.

\section{Acknowledgements}

This study was supported by a grant from the Australian Government Department of Health and was completed using data collected through the 45 and Up Study (www. saxinstitute.org.au). The 45 and Up Study is managed by the Sax Institute in collaboration with major partner 
Cancer Council NSW; and partners: the Heart Foundation; NSW Ministry of Health; NSW Department of Communities and Justice; and Australian Red Cross Lifeblood. We thank the many thousands of people participating in the 45 and Up Study.

The authors also acknowledge the other project investigators - Lily O'Donoughue Jenkins, Karl Skewes, Christine Phillips, Elizabeth Sturgiss and Raymond Lovett, who contributed to the conception, design and interpretation of the study - and the National Heart Foundation for its feedback on preliminary findings.

\section{Peer review and provenance}

Externally peer reviewed, not commissioned.

\section{Competing interests}

None declared.

\section{Author contributions}

$\mathrm{DB}, \mathrm{JA}, \mathrm{EP}, \mathrm{RK}, \mathrm{KD}, \mathrm{VW}$ and $\mathrm{EB}$ conceived and designed the analysis and drafted the manuscript. DB completed data analysis. All authors revised the work for intellectual content and approved the final version of the manuscript.

\section{References}

1. Australian Institute of Health and Welfare. The health and welfare of Australia's Aboriginal and Torres Strait Islander peoples 2015. Canberra: AlHW; 2015 [cited 2021 Jan 6]. Available from: www.aihw.gov.au/reports/indigenoushealth-welfare/indigenous-health-welfare-2015

2. King $M$, Smith A, Gracey M. Indigenous health part 2: the underlying causes of the health gap. Lancet. 2009;374(9683):76-85.

3. National Vascular Disease Prevention Alliance. Guidelines for the management of absolute cardiovascular disease risk. Canberra: NVDPA; 2012 [cited 2019 Dec 11]. Available from: informme.org.au/en/Guidelines/ Guidelines-for-the-assessment-and-management-ofabsolute-CVD-risk

4. Goff DC Jr., Lloyd-Jones DM, Bennett G, Coady S, D'Agostino RB, Gibbons R, et al. 2013 ACC/AHA guideline on the assessment of cardiovascular risk: a report of the American College of Cardiology/American Heart Association Task Force on Practice Guidelines. Circulation. 2014;129(25 Suppl 2):S49-73.

5. Calabria B, Korda RJ, Lovett RW, Fernando P, Martin T, Malamoo L, et al. Absolute cardiovascular disease risk and lipid-lowering therapy among Aboriginal and Torres Strait Islander Australians. Med J Aust. 2018;209(1):3541.
6. Peiris DP, Patel AA, Cass A, Howard MP, Tchan ML, Brady JP, et al. Cardiovascular disease risk management for Aboriginal and Torres Strait Islander peoples in primary health care settings: findings from the Kanyini Audit. Med J Aust. 2009;191(6):304-9.

7. Australian Government Department of Health. Medicare health assessment for Aboriginal and Torres Strait Islander people (MBS ITEM 715). Canberra: Australian Government; 2018 [cited 2019 Dec 11]. Available from: www1.health.gov.au/internet/main/publishing.nst/Content/ mbsprimarycare_ATSI_MBSitem715

8. Matthews V, Burgess CP, Connors C, Moore E, Peiris D, Scrimgeour D, et al. Integrated clinical decision support systems promote absolute cardiovascular risk assessment: an important primary prevention measure in aboriginal and Torres Strait Islander primary health care. Front Public Health. 2017;5:233.

9. Burgess CP, Bailie RS, Connors CM, Chenhall RD, McDermott RA, O'Dea K, et al. Early identification and preventive care for elevated cardiovascular disease risk within a remote Australian Aboriginal primary health care service. BMC Health Serv Res. 2011;11:24.

10. Krogsboll LT, Jorgensen KJ, Gotzsche PC. General health checks in adults for reducing morbidity and mortality from disease. Cochrane Database Syst Rev. 2019;1:CD009009.

11. Si S, Moss JR, Sullivan TR, Newton SS, Stocks NP. Effectiveness of general practice-based health checks: a systematic review and meta-analysis. $\mathrm{Br} \mathrm{J}$ Gen Pract. 2014;64(618):e47-53.

12. Australian Institute of Health and Welfare. Indigenous health checks and follow-ups. Canberra: AlHW; 2019 [cited 2019 Dec 11]. Available from: www.aihw.gov.au/ reports/indigenous-australians/indigenous-health-checksfollow-ups.

13. Australian Government Department of Health. National Aboriginal and Torres Strait Islander health plan 20132023. Canberra: Australian Government; 2013 [cited 2021 Jan 5]. Available from: www1.health.gov.au/internet/ main/publishing.nsf/content/B92E980680486C3BCA257B F0001BAF01/\$File/health-plan.pdf

14. Bailie C, Matthews V, Bailie J, Burgess P, Copley K, Kennedy $C$, et al. Determinants and gaps in preventive care delivery for Indigenous Australians: a crosssectional analysis. Front Public Health. 2016;4:34.

15. Bailie RS, Si D, Connors CM, Kwedza R, O'Donoghue L, Kennedy $\mathrm{C}$, et al. Variation in quality of preventive care for well adults in Indigenous community health centres in Australia. BMC Health Serv Res. 2011;11(1):139.

16. Australian Bureau of Statistics. Census of population and housing: reflecting Australia - stories from the census, 2016. Canberra: ABS; 2017 [cited 2021 Jan 5]. Available from: www.abs.gov.au/ausstats/abs@.nsf/Lookup/by\%20 Subject/2071.0 2016 Main\%20Features Aboriginal\%20 and\%20Torres\%20Strait\%20lslander\%20Population\%20 Data\%20Summary 10 
17. 45 and Up Study Collaborators. Cohort profile: the 45 and Up study. Int J Epidemiol. 2008;37(5):941-7.

18. Mealing NM, Banks E, Jorm LR, Steel DG, Clements MS, Rogers KD. Investigation of relative risk estimates from studies of the same population with contrasting response rates and designs. BMC Med Res Methodol. 2010;10:26.

19. NSW Centre for Health Record Linkage. NSW: CHeReL; 2018 [cited 2019 Dec 11]. Available from: www.cherel.org. $\mathrm{au}$

20. Australian Institute of Health and Welfare. Data linkage. Canberra: AlHW; 2018 [cited 2019 Dec 11]. Available from: www.aihw.gov.au/our-services/data-linkage

21. Australian Bureau of Statistics. Australian Statistical Geography Standard (ASGS): Volume 5 - remoteness structure, July 2016. Canberra: ABS; 2018 [cited 2019 Dec 11]. Available from: www.abs.gov.au/AUSSTATS/ abs@.nsf/Lookup/1270.0.55.005Main+Features1July\%20 2016?OpenDocument

22. Australian Institute of Health and Welfare. The active Australia survey: a guide and manual for implementation, analysis and reporting. Canberra: AlHW; 2003 [cited 2021 Jan 6]. Available from: www.aihw.gov.au/reports/physicalactivity/active-australia-survey/formats

23. Australian Institute of Health and Welfare. Aboriginal and Torres Strait Islander Health Performance Framework 2017 report: New South Wales. Canberra: AlHW; 2017 [cited 2021 Jan 5]. Available from: www.aihw.gov.au/ getmedia/580054e1-f967-4d48-82c9-bf67fbfb08d1/aihwinw-182-nsw.pdf.aspx?inline=true

24. Bailie R, Griffin J, Kelaher M, McNeair T, Percival N, Laycock $A$, et al. Sentinel sites evaluation. Final report. Canberra: Commonwealth of Australia; 2013 [cited 2021 Jan 6]. Available from: www.menzies.edu.au/icms_ docs/189996_Sentinel_Sites_Evaluation_Final_Report.pdf
25. Australian Institute of Health and Welfare. Regional variation in uptake of Indigenous health checks and in preventable hospitalisations and deaths. Canberra: AlHW; 2019 [cited 2019 Dec 11]. Available from: www.aihw.gov. au/reports/indigenous-australians/indigenous-healthchecks-hospitalisations-deaths/contents/table-of-contents

26. Dryden R, Williams B, McCowan C, Themessl-Huber M. What do we know about who does and does not attend general health checks? Findings from a narrative scoping review. BMC Public Health. 2012;12(1):723.

27. Jennings W, Spurling GK, Askew DA. Yarning about health checks: barriers and enablers in an urban Aboriginal medical service. Aust J Prim Health. 2014;20(2):151-7.

28. Schutze H, Pulver LJ, Harris M. The uptake of Aboriginal and Torres Strait Islander health assessments fails to improve in some areas. Aust Fam Physician. 2016;45(6):415-20.

29. Royal Australian College of General Practitioners, National Aboriginal Community Controlled Health Organisation. Useful high-quality MBS item 715 health checks for Aboriginal and Torres Strait Islander people: Melbourne: RACGP \& NACCHO; 2019 [cited 2019 Dec 19]. Available from: www.racgp.org.au/FSDEDEV/media/documents/ Faculties/ATSI/NACCHO-RACGP-resource-high-quality715-health-check.pdf

30. Dutton T, Stevens W, Newman J. Health assessments for Indigenous Australians at Orange Aboriginal Medical Service: health problems identified and subsequent follow up. Aust J Prim Health. 2016;22(3):233-8.

\section{Copyright: (C) $(1)$}

(C) 2021 Butler et al. This article is licensed under the Creative Commons Attribution-NonCommercial-ShareAlike 4.0 International Licence, which allows others to redistribute, adapt and share this work non-commercially provided they attribute the work and any adapted version of it is distributed under the same Creative Commons licence terms. See: www.creativecommons.org/licenses/by-nc-sa/4.0/ 